\title{
Mechanical performance of a commercial knowledge-based VMAT planning for prostate cancer
}

\author{
Mikoto Tamura ${ }^{1}$, Hajime Monzen ${ }^{1 *}$, Kenji Matsumoto ${ }^{1}$, Kazuki Kubo ${ }^{1}$, Masakazu Otsuka ${ }^{1}$, Masahiro Inada ${ }^{2}$, \\ Hiroshi Doi ${ }^{2}$, Kazuki Ishikawa², Kiyoshi Nakamatsu², Iori Sumida ${ }^{3}$, Hirokazu Mizuno ${ }^{3}$, Do-Kun Yoon ${ }^{4}$ \\ and Yasumasa Nishimura ${ }^{2}$
}

\begin{abstract}
Background: This study clarified the mechanical performance of volumetric modulated arc therapy (VMAT) plans for prostate cancer generated with a commercial knowledge-based treatment planning (KBP) and whether KBP system could be applied clinically without any major problems with mechanical performance.

Methods: Thirty consecutive prostate cancer patients who underwent VMAT using extant clinical plans were evaluated. The mechanical performance and dosimetric accuracy of the single optimized KBPs, which were trained with other 51 clinical plans, were compared with the clinical plans. The mechanical performance metrics were mean field area (MFA), mean asymmetry distance (MAD), cross-axis score (CAS), closed leaf score (CLS), small aperture score $(\mathrm{SAS})$, leaf travel (LT), modulation complexity score $\left(M C S_{v}\right)$, and monitor unit $(\mathrm{MU})$. The $\gamma$ passing rates were evaluated with ArcCheck and EBT3 film.

Results: The mean mechanical performance metrics (clinical plan vs. KBP) were as follows: $18.28 \mathrm{~cm}^{2}$ vs. $17.25 \mathrm{~cm}^{2}$ (MFA), $21.08 \mathrm{~mm}$ vs. $20.47 \mathrm{~mm}$ (MAD), 0.54 vs. 0.55 (CAS), 0.040 vs. 0.051 (CLS), 0.20 vs. 0.23 (SAS ${ }_{5 \mathrm{~mm}}$ ), $458.5 \mathrm{~mm}$ vs. $418.8 \mathrm{~mm}$ $(\mathrm{LT}), 0.27$ vs. $0.27\left(\mathrm{MCS}_{\mathrm{v}}\right)$, and 618.2 vs. 622.1 (MU), respectively. Significant differences were observed for CLS and LT. The average $\mathrm{y}$ passing rates (clinical plan vs. KBP) were as follows: $99.0 \%$ vs. $99.1 \%$ (3\%/3 mm) and $92.4 \%$ vs. $92.5 \%$ (2\%/2 mm) with ArcCHeck, and $99.5 \%$ vs. 99.4\% (3\%/3 mm) and 95.2\% vs. 95.4\% (2\%/2 mm) with EBT3 film, respectively.
\end{abstract}

Conclusions: The KBP used lower multileaf collimator (MLC) travel and more closed or small MLC apertures than the clinical plan. The KBP system of VMAT for the prostate cancer was acceptable for clinical use without any major problems.

Keywords: Knowledge-based treatment planning, Prostate cancer, Mechanical performance, Dosimetric accuracy

\section{Background}

In intensity-modulated radiotherapy (IMRT) and volumetric modulated arc therapy (VMAT), variations in knowledge and experience can lead to significant differences in the plan quality and may compromise the gains of high-precision radiotherapy $[1,2]$. Knowledge-based treatment planning (KBP) is an approach to reduce the differences in plan quality and improve planning consistency [1]. A commercial KBP module, RapidPlan (Varian Medical Systems, Palo Alto CA, USA) has been released for the

\footnotetext{
* Correspondence: hmon@med.kindai.ac.jp

${ }^{1}$ Department of Medical Physics, Graduate School of Medical Science, Kindai University, 377-2, Ohno-Higashi, Osakasayama, Osaka 589-8511, Japan Full list of author information is available at the end of the article
}

Eclipse (Varian) treatment planning system (TPS). KBP uses a statistical model generated from a library of clinically accepted plans with consistent high quality to train dose volume histograms (DVHs) [1, 3]. This model predicts an achievable DVH range and generates dose volume objectives for IMRT and VMAT plan optimization $[1,3]$.

Many studies have reported the KBP can generate the better or comparable dosimetric results in some anatomical sites [1-9]. Hussein et al. described the KBP was able to produce IMRT and VMAT treatment plans for prostate cancer with a single optimization, with sparing and conformity better than or comparable to a clinical plan [1]. In addition, Ueda et al. suggested the KBP could also reproduce the dose distributions based on the 
experience of institutions in the case of sharing the KBP model, although it was necessary to verify the registered DVH curves could match the plan design of each institution [10]. However, Kubo et al. found that KBP may encounter difficulties with high numbers of MUs and high modulation complexity [11]. At this time, we focused on the mechanical performance of the KBP, which has not been delineated. Additionally, dosimetric accuracy can be affected by mechanical performance [12, 13]. VMAT delivery requires extremely precise mechanical performance for a linear accelerator (Linac) due to the simultaneous gantry speed, dose rate, and multileaf collimator (MLC) aperture shape variations; this makes patient-specific quality assurance (QA) an essential prerequisite for actual patient treatment $[13,14]$. The purpose of this study was to clarify the mechanical performance of the VMAT plans generated by the KBP for prostate cancer and verify patient-specific QA to assess KBP's appropriateness in the clinical setting.

\section{Methods}

\section{Clinical plan and KBP}

The cases of 30 consecutive prostate cancer patients (between September 2016 and March 2017) who underwent VMAT with clinical plans were analyzed. We trained the KBP system with the other consecutive 51 cases of T1-T2c prostate cancer treated between August 2015 and August 2016. Written informed consent was obtained from all patients and the Institutional Ethics Committee approved this study (institutional review board number: 29-133). The KBP model configuration and training process is well-explained in the literature $[5,7,11]$. The clinical target volume (CTV) was defined as the prostate and seminal vesicle and delineated by experienced radiation oncologists. The planning target volume (PTV) was defined with a $6 \mathrm{~mm}$ posterior margin and a $10 \mathrm{~mm}$ margin in all other directions added to the CTV to reduce the dose at the prostate-rectal interface. The organ at risks (OARs) were rectal wall and bladder wall. The rectum was delineated up to $1.0 \mathrm{~cm}$ above and below the PTV. The rectal wall and bladder wall were delineated $4.0 \mathrm{~mm}$ inside the outer surface of rectum and bladder, respectively. The prescribed dose was 78 Gy in 39 fractions to $95 \%$ of the volume of the PTV minus rectum (PTV - R) using $10 \mathrm{MV}$ photon beams and 2 full arcs VMAT (Gantry angle: $181^{\circ}-179^{\circ}$, clockwise and $179^{\circ}-181^{\circ}$, counterclockwise, collimator angles: $30^{\circ}$ and $330^{\circ}$ ). The control point spacing was $2^{\circ}$ angular separation. All VMAT plans were calculated with the Varian Analytic Anisotropic Algorithm (AAA) [15] using the Eclipse treatment planning system ver. 13.6 for a TrueBeam (Varian) with a Millennium 120 MLC. The goals of the treatment plan in our institution were as follows: The maximum dose $\left(D_{\max }\right)$ was $<110 \%$ and the mean dose $\left(D_{\text {mean }}\right)$ was $99-103 \%$ of the prescribed dose for the PTV - R; $\mathrm{V}_{40} \mathrm{~Gy}<60 \%, \mathrm{~V}_{60}$ Gy $<35 \%, \mathrm{~V}_{70}$ Gy $<25 \%$, and $\mathrm{V}_{78 \text { Gy }}<1 \%$ for the rectal wall; $\mathrm{V}_{40 \text { Gy }}<60 \%$ and $\mathrm{V}_{70}$ Gy $<35 \%$ for the bladder wall $[10,16]$. The overlap region between PTV and rectal wall was covered with the $90 \%$ isodose line.

The geometry and dosimetry of the PTV - R, rectum, and bladder were registered in the KBP library. In this model, the geometry or dosimetry outliers were not excluded since the removal of statistical outliers had no significant impact on establishing the model [1]. Then, we performed the dose calculation using KBP with the single optimization in the 30 consecutive clinical cases.

\section{KBP validation}

The following parameters were compared between the 30 clinical plans and the 30 KBPs:

1. The maximum $\left(D_{\max }\right)$, minimum $\left(D_{\min }\right)$, and mean dose $\left(D_{\text {mean }}\right)$ of the PTV-R volume

2. Homogeneity index (HI; defined as $100 \times\left[D_{2 \%}-D_{98 \%}\right] /$ $\left.D_{50 \%}\right)$, where $D_{98 \%}, D_{2 \%}$, and $D_{50 \%}$ are doses received by $98 \%, 2 \%$, and $50 \%$ of the PTV - R, respectively [17]

3. The $95 \%$ isodose conformity index $\left(\mathrm{Cl}_{95}\right.$; defined as $\left.V_{95 \%} / V_{P T V-R}\right)$, where $V_{95 \%}$ is volume covered by $95 \%$ of the prescribed dose (74.1 Gy) and $V_{P T V-R}$ is PTV-R volume [1]

4. Dose-volume parameters of the rectal wall: $\mathrm{V}_{40} \mathrm{~Gy}$, $\mathrm{V}_{60}$ Gy, $\mathrm{V}_{70}$ Gy, and $\mathrm{V}_{78}$ Gy

5. Dose-volume parameters of the bladder wall: $\mathrm{V}_{40} \mathrm{~Gy}$ and $\mathrm{V}_{70 \text { Gy }}$

\section{Mechanical performance metrics}

For each of the 30 clinical plans and the 30 KBPs, 8 mechanical performance metrics were verified as shown in Table 1: 1. mean field area (MFA), 2. mean asymmetry distance (MAD), 3. cross-axis score (CAS), 4. closed leaf score (CLS), 5. small aperture score (SAS) [18-20], 6. leaf travel (LT), 7. modulation complexity score for VMAT $\left(\mathrm{MCS}_{\mathrm{v}}\right)$, and 8. total MUs [12, 13].

\section{Patient-specific QA}

The gamma index evaluation for patient-specific QA has been a standard technique used to evaluate measured distributions in commercial detector systems and gafchromic film against the dose distributions predicted by TPSs [21]. We employed ArcCheck (SunNuclear, Melbourne FL, USA) as a commercial detector system for patient-specific QA. The film dosimetry was also performed. The EBT3 gafchromic film (Ashland ISP Advanced Materials, Bridgewater, NJ, USA) was inserted at the sagittal plane in center of the ArcCheck. Irradiated films were scanned using Epson ES-10000G scanner (Epson Corp., Nagano, Japan) at least $24 \mathrm{~h}$ after irradiation with a resolution of $150 \mathrm{dpi}$ from red channel. A 
Table 1 Mechanical performance metrics

\begin{tabular}{|c|c|c|c|}
\hline \multicolumn{2}{|c|}{ Mechanical performance metrics } & \multirow{2}{*}{$\begin{array}{l}\text { Abbreviation } \\
\text { MFA }\end{array}$} & \multirow{2}{*}{$\begin{array}{l}\text { Description } \\
\text { Mean of the field area weighted according to the MU at each control point }\end{array}$} \\
\hline 1 & Mean field area & & \\
\hline 2 & Mean asymmetry distance & MAD & Mean lateral distance between open MLC leaf pairs apertures and the central axis \\
\hline 3 & Cross-axis score & CAS & Proportion of MLC leaf pairs crossing the central axis within the jaw aperture \\
\hline 4 & Closed leaf score & CLS & Proportion of MLC leaf pairs entirely closed within the jaw aperture \\
\hline 5 & Small aperture score & SAS & $\begin{array}{l}\text { Proportion of open MLC leaf pairs separated by less than the given thresholds } \\
(2,5,10 \text {, and } 20 \mathrm{~mm} \text { in this study) }\end{array}$ \\
\hline 6 & Leaf travel & LT & Averaged over all in-field moving leaves \\
\hline 7 & $\begin{array}{l}\text { Modulation complexity } \\
\text { score for VMAT }\end{array}$ & $\mathrm{MCS}_{\mathrm{V}}$ & $\begin{array}{l}\text { Sum over all control points of the product of the aperture area variability (AAV), leaf sequence } \\
\text { variability (LSV), and normalized MU }\end{array}$ \\
\hline 8 & Monitor unit & MU & Sum of monitor unit value for a plan \\
\hline
\end{tabular}

calibration curve, film optical density versus dose, was determined for a range from 0 to $300 \mathrm{cGy}$. For analysis, the DoseLab ver. 6.70 (Mobius Medical Systems, TX, USA) was employed. An ionization chamber (CC01, IBA, Schwarzenbruck, Germany) was used for measurement of absorbed dose at the isocenter in the ArcCheck (source-to-axis distance $(\mathrm{SAD})=100 \mathrm{~cm}$ ). Then we evaluated the differences in dose distribution and point dose at isocenter among plans and measurements for each clinical plan and KBP. The difference of dose distribution was evaluated using the passing rate of $\gamma$ index with two tolerances in terms of dose difference (DD) and distance to agreement (DTA) $(3 \% / 3 \mathrm{~mm}$ and $2 \% / 2 \mathrm{~mm})$ with a threshold at $10 \%$.

\section{Statistical analysis}

The data are expressed as the median and interquartile ranges (first quartile, third quartile), unless otherwise indicated. The Wilcoxon signed rank test was used to compare continuous variables and trends between the KBPs and the clinical plans. All statistical analyses were performed using $\mathrm{R}$ ver. 3.4.2 (The $\mathrm{R}$ Foundation for
Statistical Computing, Vienna, Austria) and $p<0.05$ was considered statistically significant. Peason's correlation was considered weak for $r<0.4$, moderate for $0.4 \leq$ $r \leq 0.7$, and strong for $r>0.7$.

\section{Results \\ KBP validation}

Table 2 summarizes the results of comparisons between the clinical plan and the KBP of $\mathrm{D}_{\text {max }}, \mathrm{D}_{\text {min }}, \mathrm{D}_{\text {mean }}$, HI and $\mathrm{CI}_{95}$ for PTV - R, dose-volume parameters for rectal wall and bladder wall. For the PTV $-R$, the $D_{\text {min }}$ and the $\mathrm{D}_{\text {mean }}$ were comparable between the clinical plan and the KBP, and the $\mathrm{D}_{\max }$ of the KBP was significantly lower than those of the clinical plan $(p<0.01)$. The homogeneity of PTV $-\mathrm{R}$ of the KBP was significantly better than those of the clinical plan $(p=0.03)$. In addition, the PTV $-\mathrm{R}$ coverage of the KBP was more conformal as indicated by the $\mathrm{CI}_{95}$ being significantly lower than the $\mathrm{CI}_{95}$ of the clinical plan $(p<0.01)$. For the rectal wall and the bladder wall, the dose-volume parameters of the KBP were comparable to those of the clinical plan except for the $V_{78}$ Gy for the rectal wall. The

Table 2 The summary of dose-volume parameters in the clinical plan and KBP

\begin{tabular}{|c|c|c|c|c|c|}
\hline & Parameter & Clinical goal & Clinical plan & $\mathrm{KBP}$ & $p$-value \\
\hline \multirow[t]{5}{*}{ PTV - R } & $D_{\max }(\%)$ & $<110 \%$ & $106.40(105.83,107.68)$ & $105.60(105.30,106.10)$ & $<0.01$ \\
\hline & $D_{\min }(\%)$ & - & $91.55(89.88,93.98)$ & $91.75(89.63,92.90)$ & 0.33 \\
\hline & $D_{\text {mean }}(\%)$ & $99-103 \%$ & $102.20(102.00,102.68)$ & $102.05(101.93,102.20)$ & 0.07 \\
\hline & $\mathrm{HI}$ & - & $5.08(4.54,6.17)$ & $4.76(4.52,4.89)$ & 0.03 \\
\hline & $\mathrm{Cl}_{95}$ & - & $1.27(1.23,1.32)$ & $1.18(1.17,1.20)$ & $<0.01$ \\
\hline \multirow[t]{4}{*}{ Rectal wall } & $V_{40 \text { Gy }}(\%)$ & $<60 \%$ & $49.10(45.38,55.20)$ & $49.60(46.03,52.93)$ & 0.94 \\
\hline & $V_{60 \text { Gy }}(\%)$ & $<35 \%$ & $26.05(22.80,29.20)$ & $27.60(24.08,30.95)$ & 0.20 \\
\hline & $V_{70 \text { Gy }}(\%)$ & $<25 \%$ & $15.00(12.40,16.88)$ & $16.40(14.70,16.90)$ & 0.13 \\
\hline & $V_{78 \text { Gy }}(\%)$ & $<1 \%$ & $0.0045(0.00,0.040)$ & $0.28(0.18,0.47)$ & $<0.01$ \\
\hline \multirow[t]{2}{*}{ Bladder wall } & $V_{40 \text { Gy }}(\%)$ & $<60 \%$ & $40.65(34.93,47.85)$ & $38.95(27.90,52.60)$ & 0.82 \\
\hline & $V_{70 ~ G y}(\%)$ & $<35 \%$ & $22.30(17.15,27.25)$ & $21.00(15.95,27.43)$ & 0.67 \\
\hline
\end{tabular}

Abbreviations: PTV $-R$ volume of the planning target volume minus rectum, $\mathrm{HI}$ homogeneity index, $\mathrm{Cl}_{95} 95 \%$ isodose conformity index 
$\mathrm{V}_{78 \text { Gy }}$ for the rectal wall of the KBP was higher than those of the clinical plan; however, it was $<1 \%$ for all cases. The mean DVHs for the clinical plan and the KBP were shown in Fig. 1. The KBP was able to generate better or comparable dosimetric results compared to clinical plan using our training model.

\section{Mechanical performance}

Table 3 summarizes the mechanical performance metrics of MFA, MAD, CAS, CLS, SAS (thresholds: 2, 5, 10, and $20 \mathrm{~mm}$ ), LT, $\mathrm{MCS}_{\mathrm{v}}$, and MU for the clinical plan and the KBP. The MFA, MAD, CAS, $\mathrm{MCS}_{\mathrm{v}}$ and MU of the KBP were almost equal to those of the clinical plan. On the other hand, there were significant differences for CLS $(p=0.03)$ and LT $(p<0.01)$. The KBP used more closed leaves and less MLC travel compared to the clinical plan. The SASs for each threshold of the KBP tended to be higher than those for the clinical plans, although there were no significant differences. This suggests that KBP system may use more small apertures in the MLCs than the clinical plan at each control point. In addition, the relationships between $\mathrm{MCS}_{\mathrm{v}}$ and LT, CLS, and SAS were shown in Fig. 2. The LT, CLS, and $\mathrm{SAS}_{2 \mathrm{~mm}}$ were not correlated to the $\mathrm{MCS}_{\mathrm{v}}$ in Fig. 2a-c. On the other hand, negative correlations were observed between $\mathrm{MCS}_{\mathrm{v}}$ and SASs for threshold $\geq 10 \mathrm{~mm}$ in Fig. 2e, f.

\section{Patient-specific QA}

Table 4 shows the $\gamma$ passing rate of each criterion for the clinical plan and the KBP. No significant differences were observed between the clinical plan and the KBP in the $\gamma$ passing rate with ArcCheck and EBT3 film. The $\gamma$ passing rates of the clinical plan and the KBP were > $90 \%$ for all cases for commonly used criteria of $3 \% /$ $3 \mathrm{~mm}$ [22-24]. For more stringent $2 \% / 2 \mathrm{~mm}$, the cases of the clinical plan and the KBP in which the $\gamma$ passing rates were $>90 \%$ with the ArcCheck were 24 and 25 cases in 30 cases, respectively. On the other hand, the cases of both the clinical plan and the KBP in which the $\gamma$ passing rates were $>90 \%$ with EBT3 film were 28 cases in 30 cases. No mechanical performance metrics were correlated to the $\gamma$ passing rate. The differences in absorbed dose at the isocenter for measurement compared to the TPS were $-0.40 \%(-0.86 \%, 0.023 \%)$ and $-0.29 \%(-0.50 \%,-0.083 \%)$ for the clinical plan and the $\mathrm{KBP}$, respectively $(p=0.48)$.

\section{Discussion}

In this study, we clarified the mechanical performance and evaluated the dosimetric accuracy of the VMAT generated by the KBP for prostate cancer. The KBP system can be used in clinical practice without any major problems, although the KBP created dose distributions
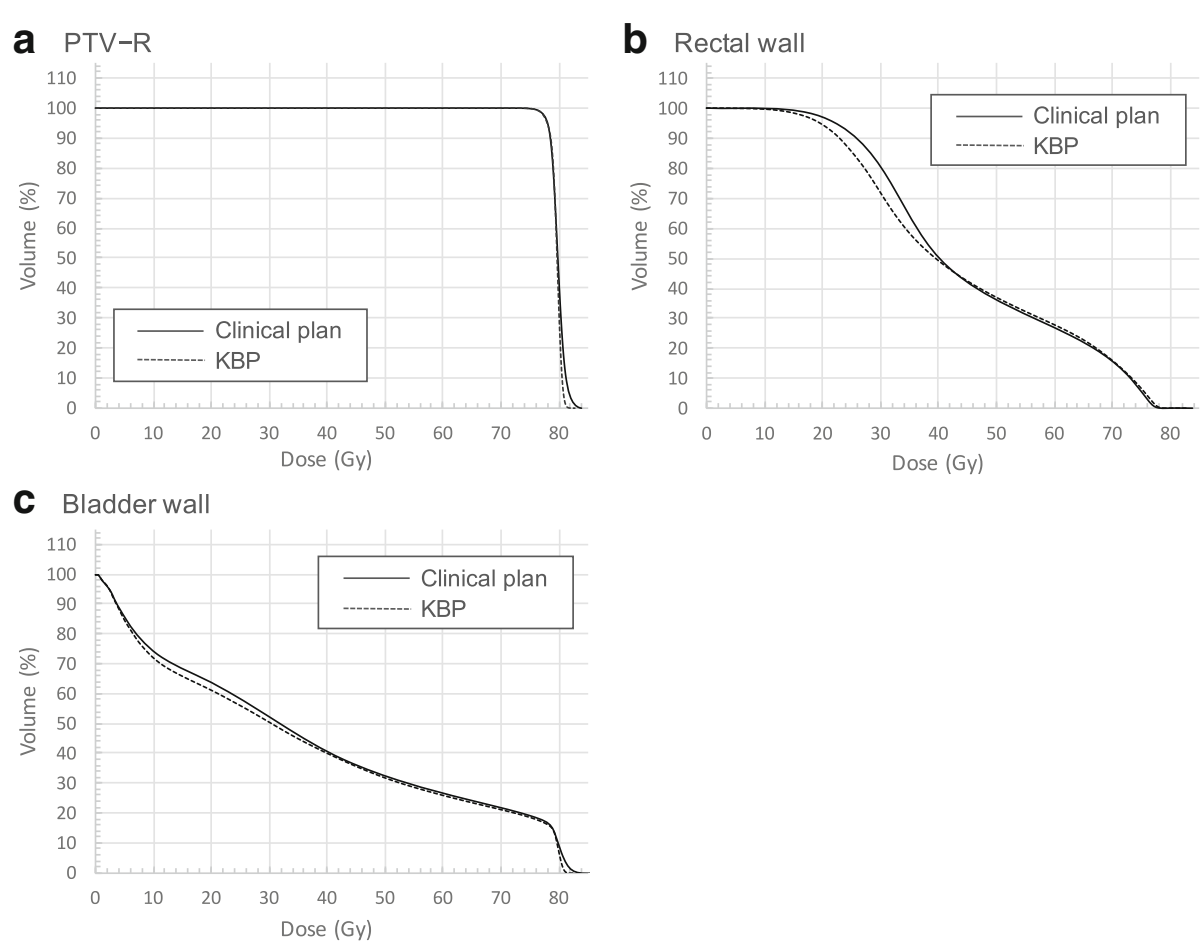

Fig. 1 The average DVHs of the clinical plan and the KBP for PTV - R (a), rectal wall (b), and bladder wall (c). For the PTV - R of the KBP, the $D_{\max }$ was lower than those of the clinical plans, which improved the homogeneity. The $V_{78}$ Gy for rectal wall of the KBP was higher than those of the clinical plans, however it was $<1 \%$ for all cases 
Table 3 The results of mechanical performance metrics for the clinical plan and KBP

\begin{tabular}{|c|c|c|c|c|c|c|c|c|c|}
\hline & \multicolumn{4}{|c|}{ Clinical Plan } & \multicolumn{4}{|l|}{$\mathrm{KBP}$} & \multirow[t]{2}{*}{$p$-value } \\
\hline & Mean & Max & Min & SD & Mean & Max & Min & SD & \\
\hline $\mathrm{MFA}\left(\mathrm{cm}^{2}\right)$ & 18.28 & 25.07 & 12.39 & 3.34 & 17.25 & 22.89 & 12.15 & 2.80 & 0.23 \\
\hline $\operatorname{MAD}(\mathrm{mm})$ & 21.08 & 25.04 & 17.27 & 2.14 & 20.47 & 24.49 & 16.88 & 1.92 & 0.38 \\
\hline CAS & 0.54 & 0.63 & 0.34 & 0.07 & 0.55 & 0.66 & 0.41 & 0.07 & 0.46 \\
\hline $\mathrm{CLS}$ & 0.040 & 0.072 & 0.0050 & 0.015 & 0.051 & 0.16 & 0.0070 & 0.027 & 0.03 \\
\hline $\mathrm{SAS}_{2 \mathrm{~mm}}$ & 0.13 & 0.22 & 0.022 & 0.042 & 0.15 & 0.24 & 0.026 & 0.044 & 0.20 \\
\hline $\mathrm{SAS}_{5 \mathrm{~mm}}$ & 0.20 & 0.30 & 0.089 & 0.053 & 0.23 & 0.33 & 0.091 & 0.087 & 0.05 \\
\hline $\mathrm{SAS}_{10 \mathrm{~mm}}$ & 0.32 & 0.48 & 0.15 & 0.071 & 0.35 & 0.48 & 0.22 & 0.063 & 0.07 \\
\hline $\mathrm{SAS}_{20 \mathrm{~mm}}$ & 0.54 & 0.71 & 0.29 & 0.087 & 0.58 & 0.77 & 0.44 & 0.081 & 0.11 \\
\hline $\mathrm{LT}(\mathrm{mm})$ & 458.51 & 550.80 & 362.80 & 41.62 & 418.81 & 499.60 & 362.10 & 29.39 & $<0.01$ \\
\hline $\mathrm{MCS}_{\mathrm{V}}$ & 0.27 & 0.31 & 0.22 & 0.023 & 0.27 & 0.30 & 0.25 & 0.015 & 0.24 \\
\hline$M U$ & 618.24 & 738.80 & 475.70 & 64.77 & 622.12 & 672.60 & 572.40 & 25.58 & 0.76 \\
\hline
\end{tabular}

Abbreviations: MFA mean field area, MAD mean asymmetry distance, $C A S$ cross-axis score, $C L S$ closed leaf score, $S A S$ small aperture score, $L T$ leaf travel, MCS modulation complexity score for VMAT, MU monitor unit

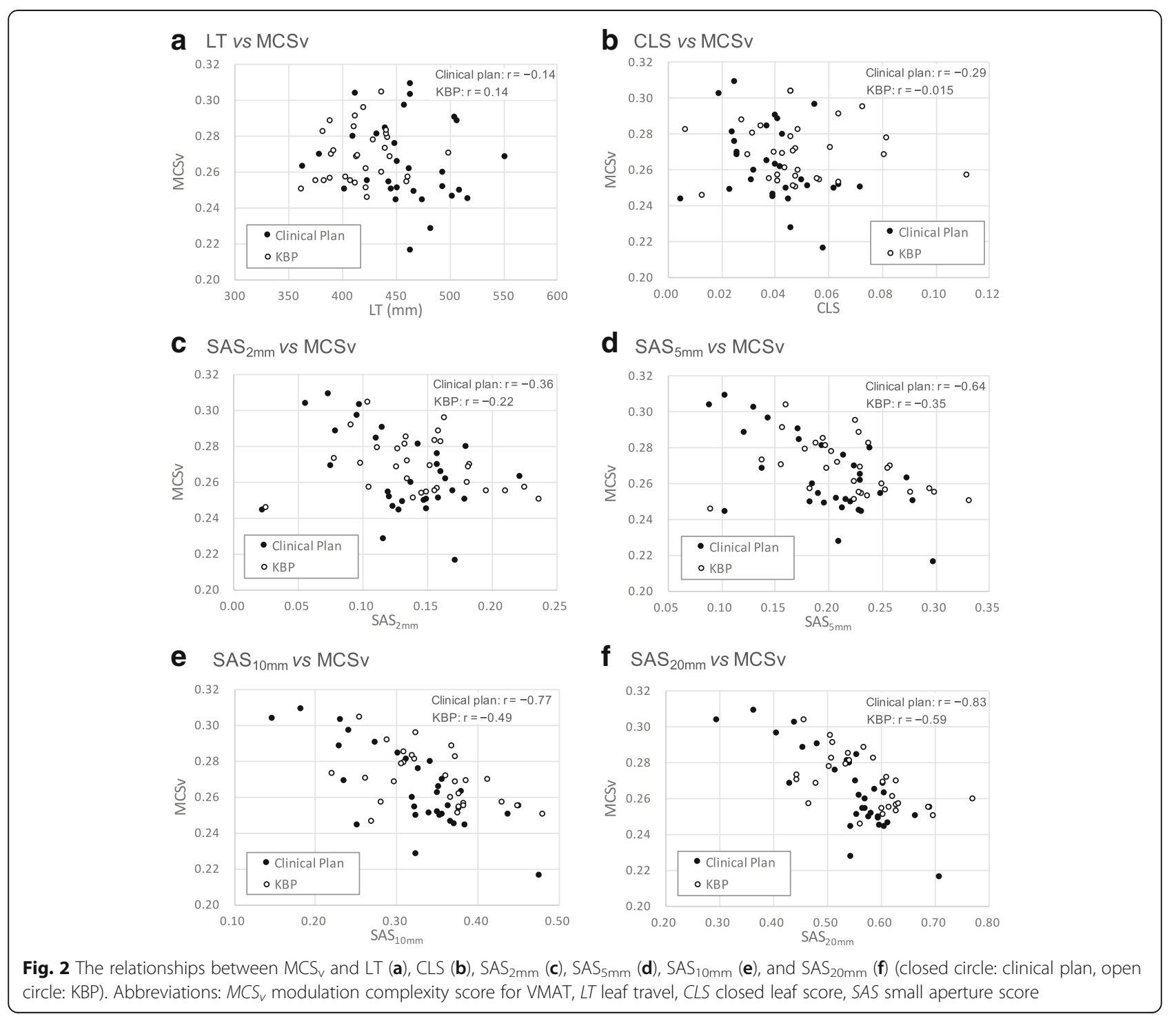


Table 4 The results of the $\gamma$ passing rate for the clinical plan and KBP with ArcCheck and EBT3 film

\begin{tabular}{|c|c|c|c|c|c|c|c|c|c|}
\hline & \multicolumn{4}{|c|}{ Clinical Plan } & \multicolumn{4}{|l|}{ KBP } & \multirow[t]{2}{*}{$p$-value } \\
\hline & Mean & Max & Min & $\overline{S D}$ & Mean & Max & Min & $\overline{\mathrm{SD}}$ & \\
\hline \multicolumn{10}{|l|}{ ArcCheck } \\
\hline $2 \% / 2 \mathrm{~mm}$ & $92.4 \%$ & $97.5 \%$ & $87.8 \%$ & 2.3 & $92.5 \%$ & $96.2 \%$ & $87.8 \%$ & 2.4 & 0.69 \\
\hline $3 \% / 3 \mathrm{~mm}$ & $99.0 \%$ & $99.8 \%$ & $97.5 \%$ & 0.6 & $99.1 \%$ & $100.0 \%$ & $97.4 \%$ & 0.7 & 0.46 \\
\hline \multicolumn{10}{|l|}{ EBT3 film } \\
\hline $2 \% / 2 \mathrm{~mm}$ & $95.2 \%$ & $99.0 \%$ & $88.7 \%$ & 2.6 & $95.4 \%$ & $99.1 \%$ & $87.3 \%$ & 3.1 & 0.65 \\
\hline $3 \% / 3 \mathrm{~mm}$ & $99.5 \%$ & $100.0 \%$ & $97.8 \%$ & 0.5 & $99.4 \%$ & $100.0 \%$ & $97.4 \%$ & 0.7 & 0.85 \\
\hline
\end{tabular}

by use of lower MLC travel and more closed or small MLC apertures compared to the clinical plan.

In the KBP model validation, Table 2 and Fig. 1 show the dose volume parameters of the KBP with the single optimization for PTV-R were better or comparable, and for rectal wall and bladder wall were comparable, to the clinical plan, respectively. The KBP also had higher homogeneity and conformity for PTV - R compared to the clinical plan. However, this model could contain a limited number of structures (e.g. femoral head, sacrum, anal canal, and bulbus penis).

In mechanical performance, the CLS and the LT values of the KBP were significantly different from the clinical plan as shown in Table 3. Additionally, the SASs for all thresholds of the KBP were higher than those of the clinical plan, although not to a significant degree. The KBP system might use lower MLC travel, and more closed or small MLC apertures at each control point than the clinical plan. The negative correlations between $\mathrm{MCS}_{\mathrm{v}}$ and SASs for threshold $\geq 10 \mathrm{~mm}$ were shown in Fig. 2e-f. The modulation complexity was increased with the proportion of small apertures increasing, which was also described by Crowe et al. [20]. Therefore, one full arc VMAT plan generated by the KBP might increase the MU and the modulation complexity compared to the clinical plan [11]. On the other hand, two full arcs VMAT plans generated by the KBP might decrease the MU and the modulation complexity. Hussein et al. also showed the MU and modulation complexity were not difference between the KBP and clinical plan for two full arcs VMAT plans [1].

The $\gamma$ passing rates for all criteria of DD and DTA were not significantly different between the clinical plan and the KBP, and all cases of the clinical plan and the $\mathrm{KBP}$ had the $\gamma$ passing rates of more than $90 \%$ for AAPM recommending criteria of $3 \% / 3 \mathrm{~mm} \mathrm{[23]} \mathrm{as}$ shown in Table 4, although the mechanical performance of the KBP did not correspond to that of the clinical plan. A patient-specific QA passed if the $\gamma$ passing rate was $>90 \%$ of points achieving the $3 \% / 3 \mathrm{~mm}$ criterion, which is acceptable in the clinical setting [22-24], although some reports describe more stringent criteria such as $2 \% / 2 \mathrm{~mm}$ [25-27]. It is necessary to understand the sensitivity and limitations of the gamma index analysis combined with the equipment in use [21].

\section{Conclusions}

The KBP with the single optimization used lower MLC travel and more closed or small MLC apertures compared to the clinical plan. The VMAT plan generated by the KBP for prostate cancer could be applied clinically without any major problems.

\begin{abstract}
Abbreviations
AAA: Analytic Anisotropic Algorithm; AAV: Aperture area variability; CAS: Cross-axis score; Cl ${ }_{95}$ : 95\% isodose conformity index; CLS: Closed leaf score; CTV: Clinical target volume; DD: Dose difference; DTA: Distance to agreement; DVH: Dose volume histogram; HI: Homogeneity index; IMRT: Intensity-modulated radiotherapy; KBP: Knowledge-based treatment planning; Linac: Linear accelerator; LSV: Leaf sequence variability; LT: Leaf travel; MAD: Mean asymmetry distance; MCSv: Modulation complexity score for VMAT; MFA: Mean field area; MLC: Multileaf collimator; MU: Monitor unit; OAR: Organ at risk; PTV - R: Volume of the PTV minus rectum; PTV: Planning target volume; QA: Quality assurance; SAD: Source-to-axis distance; SAS: Small aperture score; TPS: Treatment planning system; VMAT: Volumetric modulated arc therapy
\end{abstract}

\section{Acknowledgements}

We thank Libby Cone, MD, MA, from Edanz Group (http:// www.edanzediting.com/ac) for editing a draft of this manuscript.

\section{Funding}

This work was supported by the Japan Society for the Promotion of Science KAKENHI (grant number: 16 K10406).

\section{Availability of data and materials}

The dataset used and/or analysed during the current study are available from the corresponding author on reasonable request.

\section{Authors' contributions}

Concept and design: MT, HM. Treatment planning: MT, HM, KM, MO, MI, HD, KI, KN. Measurements: MT, KM, MO. Data analysis: MT, KK, IS, HM, DKY. Manuscript preparation: MT, HM, HD, Kl, KN, YN. All authors read and approved the final manuscript.

\section{Ethics approval and consent to participate}

This study was approved by review committee with written informed consent provided by the patients (institutional review board number: 29-133).

\section{Consent for publication}

The institutional consent form was obtained from the patients.

Competing interests

The authors declare that they have no competing interests. 


\section{Publisher's Note}

Springer Nature remains neutral with regard to jurisdictional claims in published maps and institutional affiliations.

\section{Author details}

'Department of Medical Physics, Graduate School of Medical Science, Kindai University, 377-2, Ohno-Higashi, Osakasayama, Osaka 589-8511, Japan. ${ }^{2}$ Department of Radiation Oncology, Faculty of Medicine, Kindai University, 377-2, Ohno-Higashi, Osakasayama, Osaka 589-8511, Japan. ${ }^{3}$ Department of Radiation Oncology, Graduate School of Medicine, Osaka University, 2-2, Yamada-oka, Suita, Osaka 565-0071, Japan. ${ }^{4}$ Department of Biomedical Engineering and Research Institute of Biomedical Engineering, College of Medicine, Catholic University of Korea, 505, Banpo-dong, Seocho-gu, Seoul 137-701, Korea.

Received: 23 April 2018 Accepted: 23 August 2018

Published online: 31 August 2018

\section{References}

1. Hussein M, South CP, Barry MA, Adams EJ, Jordan TJ, Stewart AJ, et al. Clinical validation and benchmarking of knowledge-based IMRT and VMAT treatment planning in pelvic anatomy. Radiother Oncol. 2016;120:473-9. https://doi.org/10.1016/j.radonc.2016.06.022

2. Tol JP, Delaney AR, Dahele M, Slotman BJ, Verbakel WFAR. Evaluation of a knowledge-based planning solution for head and neck cancer. Int J Radiat Oncol Biol Phys. 2015:91:612-20. https://doi.org/10.1016/j.jijobp.2014.11.014

3. Wu H, Jiang F, Yue H, Li S, Zhang Y. A dosimetric evaluation of knowledgebased VMAT planning with simultaneous integrated boosting for rectal cancer patients. J Appl Clin Med Phys. 2016;17:78-85. https://doi.org/10. 1120/jacmp.v17i6.6410

4. Fogliata A, Wang P-M, Belosi F, Clivio A, Nicolini G, Vanetti E, et al. Assessment of a model based optimization engine for volumetric modulated arc therapy for patients with advanced hepatocellular cancer. Radiat Oncol. 2014;9:236. https://doi.org/10.1186/s13014-014-0236-0

5. Fogliata A, Belosi F, Clivio A, Navarria P, Nicolini G, Scorsetti M, et al. On the pre-clinical validation of a commercial model-based optimisation engine: application to volumetric modulated arc therapy for patients with lung or prostate cancer. Radiother Oncol. 2014;113:385-91. https://doi.org/10.1016/j. radonc.2014.11.009

6. Fogliata A, Nicolini G, Bourgier C, Clivio A, De Rose F, Fenoglietto P, et al. Performance of a knowledge-based model for optimization of volumetric modulated arc therapy plans for single and bilateral breast irradiation. PLoS One. 2015;10:1-12. https://doi.org/10.1371/journal.pone.0145137

7. Fogliata A, Nicolini G, Clivio A, Vanetti E, Laksar S, Tozzi A, et al. A broad scope knowledge based model for optimization of VMAT in esophageal cancer: validation and assessment of plan quality among different treatment centers. Radiat Oncol. 2015;10:220. https://doi.org/10.1186/ s13014-015-0530-5

8. Snyder K, Kim J, Reding A, Fraser C, Lu S, Gordon J, et al. Development and evaluation of a knowledgebased model for treatment planning of lung cancer patients using stereotactic body radiotherapy (SBRT). J Appl Clin Med Phys. 2016;17:263-75. https://doi.org/10.1120/jacmp.v17i6.6429

9. Chang ATY, Hung AWM, Cheung FWK, Lee MCH, Chan OSH, Philips $\mathrm{H}$, et al. Comparison of planning quality and efficiency between conventional and knowledge-based algorithms in nasopharyngeal cancer patients using intensity modulated radiation therapy. Int J Radiat Oncol Biol Phys. 2016;95: 981-90. https://doi.org/10.1016/j.jirobp.2016.02.017

10. Ueda Y, Fukunaga J, Kamima T, Adachi Y, Nakamatsu K, Monzen H. Evaluation of multiple institutions' models for knowledge-based planning of volumetric modulated arc therapy (VMAT) for prostate cancer. Radiat Oncol. 2018;46:46. https://doi.org/10.1186/s13014-018-0994-1

11. Kubo K, Monzen H, Ishii K, Tamura M, Kawamorita R, Sumida I, et al. Dosimetric comparison of RapidPlan and manually optimized plans in volumetric modulated arc therapy for prostate cancer. Phys Med. 2017:44: 199-204. https://doi.org/10.1016/j.ejmp.2017.06.026

12. McNiven AL, Sharpe MB. Purdie TG. A new metric for assessing IMRT modulation complexity and plan deliverability. Med Phys. 2010;37:505-15. https://doi.org/10.1118/1.3276775

13. Masi L, Doro R, Favuzza V, Cipressi S, Livi L. Impact of plan parameters on the dosimetric accuracy of volumetric modulated arc therapy. Med Phys. 2013;40:071718. https://doi.org/10.1118/1.4810969
14. Li G, Zhang Y, Jiang X, Bai S, Peng G, Wu K, et al. Evaluation of the ArcCHECK QA system for IMRT and VMAT verification. Phys Med. 2013;29: 295-303. https://doi.org/10.1016/j.ejmp.2012.04.005

15. Van EA, Tillikainen $L$, Huyskens DP, Tenhunen $M$, Helminen $H$, Siljämaki $S$, et al. Testing of the analytical anisotropic algorithm for photon dose calculation. Med Phys. 2006:33:4130-48. https://doi.org/10.1118/1.2358333

16. Hirose $Y$, Nakamura M, Tomita T, Kitsuda K, Notogawa T, Miki K, et al. Evaluation of different set-up error corrections on dose-volume metrics in prostate IMRT using CBCT images. J Radiat Res. 2014;55:966-75. https://doi. org/10.1093/jrr/rru033

17. Wu H, Jiang F, Yue $H$, Zhang $H$, Wang $K$, Zhang Y. Applying a RapidPlan model trained on a technique and orientation to another : a feasibility and dosimetric evaluation. Radiat Oncol. 2016;11:108. https://doi.org/10.1186/ s13014-016-0684-9

18. Crowe S. Treatment plan complexity metrics for predicting IMRT pretreatment quality assurance results. Australas Phys Eng Sci Med. 2014;37: 475-82. https://doi.org/10.1007/s13246-014-0274-9

19. Kairn T, Crowe SB, Kenny J, Knight RT, Trapp JV. Predicting the likelihood of QA failure using treatment plan accuracy metrics. J Phys Conf Ser. 2014;489: 012051. https://doi.org/10.1088/1742-6596/489/1/012051

20. Crowe SB, Kairn T, Middlebrook N, Sutherland B, Hill B, Kenny J, et al. Examination of the properties of IMRT and VMAT beams and evaluation against pre-treatment quality assurance results. Phys Med Biol. 2015;60: 2587-601. https://doi.org/10.1088/0031-9155/60/6/2587

21. Hussein M, Rowshanfarzad P, Ebert MA, Nisbet A, Clark CH. A comparison of the gamma index analysis in various commercial IMRTNMAT QA systems. Radiother Oncol. 2013;109:370-6. https:/doi.org/10.1016/j.radonc.2013.08.048

22. Aristophanous M, Suh Y, Chi PC, Whittlesey LJ, Laneave S, Mary K. Initial clinical experience with ArcCHECK for IMRTNMAT QA. J Appl Clin Med Phys. 2016;17:20-33. https://doi.org/10.1120/jacmp.v17i5.6118

23. Ezzell GA, Burmeister JW, Dogan N, LoSasso TJ, Mechalakos JG, Mihailidis D, et al. IMRT commissioning: multiple institution planning and dosimetry comparisons, a report from AAPM task group 119. Med Phys. 2009;36:535973. https://doi.org/10.1118/1.3238104

24. Crowe SB, Sutherland B, Wilks R, Seshadri V, Sylvander S, Trapp JV, et al. Technical note: relationships between gamma criteria and action levels: results of a multicenter audit of gamma agreement index results. Med Phys. 2016;43:1501-6. https://doi.org/10.1118/1.2710949

25. García-Vicente F, Fernández V, Bermúdez R, Gómez A, Pérez L, Zapatero A, et al. Sensitivity of a helical diode array device to delivery errors in IMRT treatment and establishment of tolerance level for pretreatment QA. J Appl Clin Med Phys. 2012;13:111-23. https://doi.org/10.1120/jacmp.v13i1.3660

26. Steers JM, Fraass BA. IMRT QA: Selecting gamma criteria based on error detection sensitivity. Med Phys. 2016:43:1982-94. https://doi.org/10.1118/1.4943953

27. Nelms BE, Opp D, Robinson J, Wolf TK, Zhang G, Moros E, et al. VMAT QA: measurement-guided 4D dose reconstruction on a patient. Med Phys. 2012; 39:4228-38. https://doi.org/10.1118/1.4729709

Ready to submit your research? Choose BMC and benefit from:

- fast, convenient online submission

- thorough peer review by experienced researchers in your field

- rapid publication on acceptance

- support for research data, including large and complex data types

- gold Open Access which fosters wider collaboration and increased citations

- maximum visibility for your research: over $100 \mathrm{M}$ website views per year

At $\mathrm{BMC}$, research is always in progress.

Learn more biomedcentral.com/submissions 